Ann. Biol. anim. Bioch. Biophys., 1978, 18 (5), 1107-1115.

\title{
Influence combinée de l'ingestion de tricaproïne et du mode de distribution des repas sur l'utilisation de l'énergie et de l'azote par le rat en croissance
}

\author{
par B. AUROUSSEAU, J. M. PEREZ, J. C. BOUVIER, M. VERMOREL \\ avec la collaboration technique de Françoise DUBOISSET et J. C. PACROT \\ Laboratoire d'Ełude du Métabolisme énergétique, I.N.R.A., \\ Theix, Saint-Genès-Champanelle, 63110 Beaumont.
}

Summary. Energy and nitrogen utilization in the growing rat: Combined effect of tricaproine intake and method of meal distribution.

$A$ « control 》 and a « tricaproine » diet, balanced at $5 \mathrm{mg} \mathrm{Nd} / \mathrm{kcal} E M$, were fed to lots of young male rats between 90 and $190 \mathrm{~g}$ liveweight. The diets were offered either in one single meal or in 4 meals per day. Feeding the rations in 4 meals caused a highly significant reduction (13 p. 100) of energy fixation and a slight increase in nitrogen fixation $(+1.5 \mathrm{p} .100)$ in the control group. This treatment hardly changed the overall utilization of the tricaproine diet (0.5 and 2.7 p. 100 less energy and nitrogen fixed, respectively), but increased the amount of nitrogen and energy fixed in the paw muscles with both diets. As compared to the homologous control treatment, the tricaproine ration induced a marked reduction in energy $(-7$ p. 100) and nitrogen $(-2.3$ p. 100) fixation when the diets were offered in a single meal ; it led to a relative increase of fixed energy $(+6$ p. 100) and to a lower nitrogen fixation ( -6.5 p. 100) when 4 meals per day were given.

Un premier essai nous a permis de mettre en évidence que, chez le Rat en croissance, l'ingestion d'acide caproïque, en substitution isoénergétique à une partie de l'amidon d'un régime témoin, améliore à la fois l'utilisation des matières azotées et de l'énergie ingérée (Chenat ef al., 1976).

Pour chercher à analyser quels sont les mécanismes qui se succèdent, au niveau du métabolisme intermédiaire, pour aboutir aux effets globaux observés au niveau de la croissance des animaux, nous nous sommes intéressés à mieux standardiser les conditions expérimentales ef l'état nutritionnel des animaux au moment des diverses analyses. La maîtrise du rythme alimentaire semble répondre à ces préoccupations : cette technique, comparée à celle de la libre consommation, permet en effet, dans le domaine de l'étude du métabolisme des acides aminés en particulier, l'obtention de résultats plus homogènes.

Cependant, des rats en croissance recevant des régimes à base d'amidon et de farine de poisson et ingérant leur ration quotidienne en 4 ou 6 repas régulièrement 
espacés dans le temps fixent dans leurs tissus, pour un même niveau d'ingestion, davantage de protéines et moins d'énergie et de lipides que les animaux en libre consommation (Bouvier et al., 1971). Il était donc nécessaire d'étudier d'abord l'influence combinée de l'ingestion d'acide caproïque et du mode de distribution de la ration sur la croissance du rat et sur l'utilisation de l'énergie ef de l'azote qu'il ingère.

\section{Matériel et méthodes.}

Quatre-vingt rats mâles de souche Wistar sevrés au poids de $50 \mathrm{~g}$ ont ensuite reçu des aliments concentrés agglomérés du commerce avant d'être répartis en 5 lots homologues au poids de $90 \mathrm{~g}$. Un de ces lots a été sacrifié en début d'expérience et les autres ont été affectés à quatre traitements différents : 2 régimes expérimentaux (《Témoin 》) et «Tricaproïne » = «Tri C6 »), et 2 rythmes alimentaires (" une distribution » ou «quatre repas » par jour).

Les régimes expérimentaux semi-synthétiques (tabl. 1) à base d'amidon et de farine de hareng de Norvège étaient équilibrés au taux de $5 \mathrm{mg}$ d'azote digestible par kilocalorie d'énergie métabolisable ( $\mathrm{mg} \mathrm{Nd/kcal} \mathrm{EM).} \mathrm{Ils} \mathrm{étaient} \mathrm{supplémentés} \mathrm{en}$ acides aminés indispensables selon les besoins du rat en croissance définis par Pawlak et Pion $(1968,1971)$ et comportaient, en outre 1,6 p. 100 d'huile de maïs (pour la couverture des besoins en acides gras essentiels), 1,6 p. 100 d'huile de palme, 10 p. 100 de saccharose et 3,5 p. 100 de mélange minéral et vitaminique. Pour constituer le régime

\section{TABLEAU 1}

Composition et utilisation digestive ef métabolique des régimes expérimentoux (g pour $1000 \mathrm{~g}$ d'aliment)

\begin{tabular}{|c|c|c|}
\hline & Témoin & Tricaproïne \\
\hline 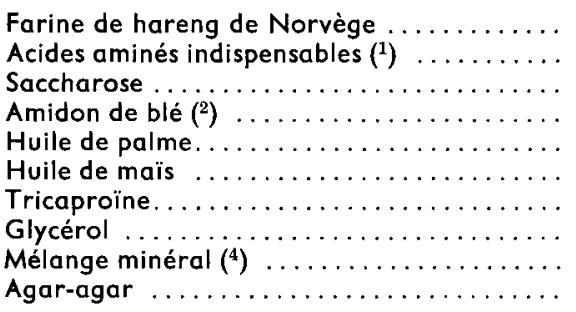 & $\begin{array}{r}174,0 \\
6,8 \\
96,0 \\
628,7 \\
16,4 \\
16,4 \\
- \\
12,3 \\
32,8 \\
16,4\end{array}$ & $\begin{array}{r}186,9 \\
7,4 \\
104,2 \\
516,3 \\
17,8 \\
17,8 \\
96,1 \\
-1 \\
35,6 \\
17,8\end{array}$ \\
\hline 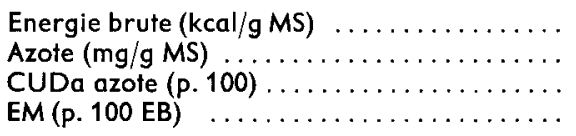 & $\begin{array}{l}4,368 \\
23,22 \\
87,6 \\
92\end{array}$ & $\begin{array}{l}4,768 \\
24,72 \\
86,6 \\
91,7\end{array}$ \\
\hline Azote $(\mathrm{mg} \mathrm{Nd} / \mathrm{kcal} E M) \ldots \ldots \ldots \ldots$ & 5,06 & 4,90 \\
\hline
\end{tabular}

(1) Composition du mélange : DL méthionine (40 p. 100), L phénylalanine (24,6 p. 100), L lysine $\mathrm{HCl}(12,3$ p. 100), $L$ tryptophane (12,3 p. 100), $L$ thréonine $(5,5$ p. 100), $L$ isoleucine (5,5 p. 100).

${ }^{(2)}$ Le mélange vitaminique est inclu à une partie de l'amidon du régime. ef $D$.

(3) L'huile de maïs assure les apports en acides gras essentiels ef est supplémentée en vitamines A

${ }^{4}$ ) Mélange minéral selon Pawlak ef Pion (1968). 
"Tri C6 », l'acide caproïque était substitué, en quantité isoénergétique, à une partie de l'amidon du régime témoin (15 p. 100 en énergie) et introduit dans le régime expérimental sous forme de tricaproïne, les apports d'énergie par le glycérol étant semblables dans les deux régimes.

Les distributions étaient ajustées chaque jour en vue d'obtenir des ingestions d'EM voisines pour chacun des 4 lots. Les rations ont été distribuées en une seule fois ou en quatre repas égaux régulièrement espacés au cours de la journée (1 repas toutes les $6 \mathrm{~h}$ ) à l'aide d'un dispositif automatique de seringues motorisées.

L'utilisation digestive et métabolique des aliments a été déterminée sur des groupes de 6 animaux pendant une période de 10 jours après une adaptation de 5 jours aux régimes et au maintien en cage à métabolisme. L'expérience s'est déroulée sur une période de 16 jours. Ensuite les animaux ont été sacrifiés; on a vidé leur tube digestif et procédé au prélèvement des muscles d'une patte postérieure sur la carcasse de chaque animal. Les carcasses et les muscles des pattes ont été conservés à $-15^{\circ} \mathrm{C}$ puis broyés et homogénéisés par lot entier, selon le schéma décrit antérieurement (Aurousseau, 1972). Les dosages de matière sèche (48 h à l'étuve à $80^{\circ} \mathrm{C}$ ), d'énergie (calorimètre adiabatique Gallenkamp) ef d'azote (méthode macro Kjeldahl) ont été effectués en trois exemplaires sur deux échantillons différents représentatifs du lot entier.

Pour l'analyse statistique des résultats, nous avons admis, comme précédemment (Aurousseau, 1972), un coefficient de variation de la composition des carcasses des animaux d'un même lot égal à 6 p. 100. Enfin, pour faciliter l'interprétation des résultats, les bilans d'énergie ef d'azote ont été corrigés pour les différences de EM ingérée sur la base de rendements déterminés antérieurement en chambre respiratoire (Vermorel et Keller, 1967; Chenat et al., 1976). Nous avons également utilisé la notion d'efficacité de l'utilisation de l'énergie de l'acide caproïque comparée à celle de l'amidon (Aurousseau ef al., 1970), qui correspond au rapport entre les quantités de EM d'amidon ef d'acide caproïque qui, apportées en substitution l'une de l'autre, auraient permis une fixation d'énergie comparable.

\section{Résultats.}

Les coefficients d'utilisation digestive de l'énergie et de l'azote ingérés ont été respectivement de 93,3 p. 100 et de 87,6 p. 100 dans le cas du régime "Témoin » et de 92,3 p. 100 et de 86,6 p. 100 dans le cas du régime «Tri C6 ». La proportion de EM a été respectivement de 90,2 p. 100 ou de 89,3 p. 100 d'énergie brute $(E B)$ pour chacun des régimes. Aucune différence n'a atteint le seuil de signification.

Des vitesses de croissance élevées $(6,3 \mathrm{~g} / \mathrm{j}$, du même ordre que celles observées précédemment pour le même type d'expérimentation (Bouvier et al., 1971) ont été obtenues. Les animaux recevant le régime témoin en " une distribution » ont dû être restreints pour que les niveaux d'ingestion soient comparables d'un lot à l'autre, les 3 autres lots présentant spontanément des niveaux d'ingestion voisins. Les différences de vitesse de croissance ou de poids vif vide des animaux en fin d'expérience ont été minimes et n'ont pas atteint le seuil de signification. Au niveau de la teneur des carcasses en matière sèche : 34,0-32,6-33,1 et 34,0 p. 100 respectivement pour les animaux 
"Témoins », « Témoins 4 repas par jour », " Tricaproïne » et « Tricaproïne 4 repas par jour ", les différences ont été un peu plus marquées, mais elles sont restées non significatives.

Des différences importantes sont apparues, en ce qui concerne l'utilisation de l'énergie et de l'azote ingérés (tabl. 2 et 3 ). Ainsi, la distribution du régime témoin en 4 repas égaux a conduit, au niveau de la carcasse entière, à une réduction hautement significative de 13 p. 100 des quantités d'énergie fixée, plus importante que celle observée précédemment ( 9 p. 100). Par contre, les quantités d'azote fixé n'ont pas été significativement augmentées ( $+1,6$ p. 100) alors qu'elles l'avaient été $(+6,3$ p. 100) au cours des essais précédents (Bouvier et al., 1971). Cependant, le poids des muscles des pattes postérieures ainsi que leurs contenus en énergie et en azote ont été accrus de 6 à 7 p. 100 par la distribution des rations en 4 repas par jour. Dans ces muscles, le contenu en protéines représentait $74 p$. 100 de l'énergie totale, et son accroissement consécutivement au traitement « 4 repas » a été de 75 p. 100 de l'accroissement de l'énergie totale.

\section{TABLEAU 2}

Influence du rythme de distribution des repas ef de l'ingestion d'acide caproïque sur la croissance ef l'utilisation de l'azote ingéré chez le rat en croissance pendant une période expérimentale de 16 jours (valeurs moyennes et écart-type par animal pour la période)

\begin{tabular}{|c|c|c|c|c|}
\hline \multirow{2}{*}{$\begin{array}{c}\text { Régimes expérimentaux } \\
\begin{array}{c}\text { Rythme de distribution } \\
\text { des repas }\end{array}\end{array}$} & \multicolumn{2}{|c|}{ Régime témoin } & \multicolumn{2}{|c|}{ Régime Tricaproïne (Tri C6) } \\
\hline & $\begin{array}{l}\text { une distri- } \\
\text { bution }\end{array}$ & $\begin{array}{l}4 \text { repas } \\
\text { par jour }\end{array}$ & $\begin{array}{l}\text { une distri- } \\
\text { bution }\end{array}$ & $\begin{array}{l}4 \text { repas } \\
\text { par jour }\end{array}$ \\
\hline $\begin{array}{l}\text { Nombre d'animaux } \ldots \ldots \\
\text { Poids initial }(g) \ldots \ldots \ldots \\
\text { Poids final }(g) \ldots \ldots \ldots \ldots \\
\text { Vitesse de croissance }(\mathrm{g} / \mathrm{j}) \\
\text { Poids vide à l'abatt. }(\mathrm{g}) \ldots \ldots \\
\text { Muscles des pattes }(\mathrm{g}) \ldots \ldots \\
\text { EM ingérée }(\mathrm{kcal}) \ldots \ldots \ldots \\
\text { Em ingérée }(\mathrm{MJ}) \ldots \ldots \ldots \ldots \\
\text { Azote fixé }(\mathrm{g}) \ldots \ldots \\
\text { Azote fixé corrigé }(\mathrm{g}) \ldots \ldots \\
\text { Azote fixé corrigé }(\mathrm{p} .100) \\
\text { Azote fixé corrigé }(\mathrm{mg} / \mathrm{j}) \ldots \\
\text { Azote des muscles des pattes } \\
\text { (mg/animal) ............. }\end{array}$ & $\begin{array}{c}16 \\
91,2 \pm 1,2 \\
191,9 \pm 1,4 \\
6,29 \pm 0,14 \\
182,5 \pm 0,8 \\
9,67 \pm 0,14 \\
928,1 \\
3,883 \\
2,889 \\
2,889 b \\
100 \\
180,6\end{array}$ & $\begin{array}{c}16 \\
91,7 \pm 1,2 \\
192,6 \pm 2,1 \\
6,31 \pm 0,15 \\
179,9 \pm 0,6 \\
10,35 \pm 0,21 \\
928,1 \\
3,883 \\
2,934 \\
2,934^{b} \\
101,6 \\
183,4\end{array}$ & $\begin{array}{c}17 \\
91,9 \pm 1,2 \\
189,9 \pm 1,5 \\
6,13 \pm 0,13 \\
181,0 \pm 0,4 \\
9,63 \pm 0,17 \\
930,3 \\
3,891 \\
2,835 \\
2,793 a b \\
97,7 \\
174,6\end{array}$ & $\begin{array}{c}15 \\
92,2 \pm 0,8 \\
193,5 \pm 2,1 \\
6,33 \pm 0,12 \\
181,5 \pm 2,1 \\
10,04 \pm 0,25 \\
943,3 \\
3,946 \\
2,791 \\
2,744^{a} \\
95 \\
171,5\end{array}$ \\
\hline
\end{tabular}

Les valeurs significativement différentes $(P<0,05)$ sont signalées par des exposants différents.

* Correction pour les différences de EM ingérée, la variation d'azote fixé (en énergie) étant estimée à 11 p. 100 de la variation de EM ingérée (Chenat et al., 1976).

Par rapport aux animaux « Témoins » homologues, ceux qui ont ingéré le régime «Tri C6 » en libre consommation ont présenté, à EM ingérée égale, une réduction significative des quantités d'énergie fixées ( -7 p. 100) ainsi qu'une réduction moins marquée (-2,3 p. 100) des quantités d'azote fixées. Le calcul (tabl. 3) montre que l'acide caproïque a été utilisé avec une efficacité égale à 80 p. 100 de celle de l'amidon. Ces résultats concordent avec les effets (non significatifs) observés au niveau des vitesses de 
croissance et des teneurs en matière sèche des carcasses. Ils soni opposés aux résultats antérieurs (Chenat et al., 1976). Cet effet a été également observé au niveau des muscles des pattes postérieures des animaux dont le poids ef les contenus en énergie et en azote ont été réduits de 1 p. 100 environ.

\section{TABLEAU 3}

Influence du rythme de distribution des repas ef de l'ingestion d'acide caproïque sur l'utilisation de l'énergie ingérée par le rat en croissance pendant une période expérimentale de 16 jours

(valeurs moyennes par animal pour la période)

\begin{tabular}{|c|c|c|c|c|}
\hline \multirow{2}{*}{ Régimes expérimentaux } & \multicolumn{2}{|c|}{ Régime témoin } & \multicolumn{2}{|c|}{$\begin{array}{l}\text { Régime tricaproïne } \\
(\ll \text { Tri C6 } »)\end{array}$} \\
\hline & $\begin{array}{l}\text { une distri- } \\
\text { bution }\end{array}$ & $\begin{array}{l}4 \text { repas } \\
\text { par jour }\end{array}$ & $\begin{array}{l}\text { une distri- } \\
\text { bution }\end{array}$ & $\begin{array}{l}4 \text { repas } \\
\text { par jour }\end{array}$ \\
\hline $\begin{array}{l}\text { EM ingérée }(\mathrm{kcal}) \ldots \ldots \ldots \ldots \ldots \ldots \\
\text { EM ingérée }(\mathrm{KJ}) \ldots \ldots \ldots \ldots \\
\text { Energie fixée }(\mathrm{kcal}) \ldots \ldots \ldots \ldots \ldots \ldots \\
\text { Energie fixée }(\mathrm{KJ}) \ldots \ldots \ldots \ldots \ldots \ldots \\
\text { Energie fixée corrigée }{ }^{*}(\mathrm{kcal}) \ldots \ldots \ldots \\
\text { Energie fixée corrigée }(\mathrm{KJ}) \ldots \ldots \ldots \ldots \\
\text { Energie fixée corrigée }{ }^{*}(\mathrm{p} .100) \ldots \ldots \\
\text { Energie des muscles des pattes }(\mathrm{kcal}) \\
\text { Energie des muscles des pattes }(\mathrm{KJ})\end{array}$ & $\begin{array}{l}928,1 \\
3883 \\
274,5 \\
1148 \\
274,5 a \\
1148^{a} \\
100 \\
14,72 \\
61,6\end{array}$ & $\begin{array}{c}928,1 \\
3883 \\
239,7 \\
1003 \\
239,7^{b} \\
1003^{b} \\
87,3 \\
15,62 \\
65,3\end{array}$ & $\begin{array}{c}930,3 \\
3891 \\
257,0 \\
1075 \\
255,4^{c} \\
1068^{c} \\
93,0 \\
14,64 \\
61,2\end{array}$ & $\begin{array}{l}943,3 \\
3946 \\
264,6 \\
1107 \\
253,2 c \\
1059 c \\
92,5 \\
15,95 \\
66,7\end{array}$ \\
\hline \multicolumn{5}{|l|}{ Calculs théoriques $\left(^{1}\right)$} \\
\hline $\begin{array}{l}\text { - EM Production glucides (kcal) } \\
\text { - EM Entretien glucides (kcal).. } \\
\text { Energie de l'acide caproïque (kcal) .. } \\
\text { EM glucide correspondante (kcal) ... } \\
\text { Efficacité de l'énergie de l'acide com- } \\
\text { parée à celle de l'amidon (p. 100) .. }\end{array}$ & $\begin{array}{c}386,6 \\
541,5 \\
- \\
-\end{array}$ & $\begin{array}{l}- \\
-\end{array}$ & $\begin{array}{r}362,0 \\
541,5 \\
139,5 \\
112,7 \\
80,8\end{array}$ & $\frac{-}{-}$ \\
\hline
\end{tabular}

Les valeurs significativement différentes $(P<0,05)$ sont signalées par des lettres différentes.

* Correction par les différences de EM ingérées, le rendement d'utilisation de EM par la croissance étant estimé à 71 p. 100 (Vermorel et Keller, 1967).

(1) Calculs développés antérieurement (Aurousseau ef al., 1970).

Enfin, la distribution des rations en 4 repas par jour, n'a pas modifié de façon importante, dans le cas du régime «Tri C6 », l'utilisation globale des nutriments ingérés. Les quantités d'énergie et d'azote fixées ont été réduites de 0,5 et de 2,7 p. 100 respectivement. Le poids et le contenu en énergie el en azote des muscles des pattes ont cependant été augmentés de 4,3 p. 100, 9,0 p. 100 et 4,9 p. 100 respectivement. La part des protéines n'a représenté dans ce cas que 40 p. 100 de l'accroissement de l'énergie de ces muscles.

Dans ces conditions, par rapport aux résultats obtenus dans le cas de la distribution du régime «Témoin » en 4 repas égaux, l'ingestion d'un régime «Tri C6 » en 4 repas par jour a donc conduit, dans la carcasse entière, à une fixation d'énergie plus élevée ( +6 p. 100) et à une fixation d'azote plus faible ( $-6,5$ p. 100). Au niveau des muscles des pattes, ce traitement a eu pour effet d'en réduire le poids ( -3 p. 100) et le contenu en azote (-2,2 p. 100) et d'en accroître le contenu en énergie $(+2,1$ p. 100). 


\section{Discussion.}

La distribution du régime témoin en 4 repas par jour a conduit à des résultats sensiblement différents de ceux observés au cours d'une expérience antérieure (Bouvier ef al., 1971) : la réduction des quantités d'énergie fixée a été plus marquée ( -13 p. 100 contre -9 p. 100) et l'augmentation des quantités d'azote fixé moins marquée (+1,6 p. 100 contre $+6,3$ p. 100).

Deux phénomènes pourraient expliquer ces constatations : en premier lieu, des restrictions alimentaires ont été appliquées aux animaux en libre consommation. Selon des résultats antérieurs (Aurousseau, 1972) conformes à ceux obtenus par Mahendra et Miller (1969) mais opposés à ceux de Rider et Chow (1971), on a pu ainsi améliorer artificiellement l'utilisation de l'énergie et de l'azote ingérés.

En second lieu, nos régimes comportaient près de 9 p. 100 de l'énergie sous forme de saccharose dont l'effet a pu se manifester de façon différente selon que les animaux étaient en libre consommation ou soumis à un rythme de 4 repas par jour. Nous avons pu ainsi observer (résultats non publiés) une augmentation de la fixation d'énergie et d'azote chez des animaux en libre consommation, consécutivement au remplacement d'une partie de l'amidon d'un régime témoin par du saccharose. Ces observations sont conformes aux résultats obtenus par Mac Rae et al., (1974). Cet effet est vraisemblablement lié aux propriétés insulino-sécrétrices du saccharose et aux effets de l'insuline libérée (Horino et al., 1968 ; Wool et al., 1968 ; Grizard et al., 1974 ; Nakano et Ashida, 1975). Or, les effets de l'insuline sur le métabolisme intermédiaire peuvent s'inverser selon le contexte nutritionnel et les orientations préalables de l'activité des divers organes : cette hormone qui accélère habituellement l'utilisation des nutriments circulants et réduit la libération d'acides gras au niveau des tissus adipeux (Fain ef al., 1966) peut aussi, inversement accélérer la remobilisation des acides gras à partir de tissus adipeux antérieurement en état de lipolyse, in vitro (Chlouverakis, 1967 ; Miller et Allen, 1973) ou in vivo (Kovacev et Scow, 1966). L'introduction de saccharose dans les rations peut ainsi avoir des effets différents selon que le régime est offert en « 1 distribution » ou suivant un rythme de « 4 repas » égaux régulièrement espacés au cours des $24 \mathrm{~h}$. L'accroissement apparent de l'activité des animaux soumis à un rythme de 4 repas par jour pourrait, dans ces conditions, constituer l'origine principale de la modification des orientations du métabolisme des animaux dans le sens d'une lipolyse accrue et d'une réponse différente à l'ingestion de saccharose.

L'obtention d'effets notablement différents au niveau des muscles des pattes par rapport à celui obtenu dans la carcasse entière concorde avec ces hypothèses. En effet, l'amélioration de la fixation d'azote a été plus marquée dans les muscles des pattes. De plus, la fixation d'énergie a été à ce niveau, en augmentation, alors qu'elle a été en diminution au niveau de la carcasse entière. Cet effet n'avait pas été observé lors des essais précédents (Bouvier et al., 1971). Il pourrait correspondre aux mécanismes suivants : un effet propre de la distribution des rations en quatre repas par jour (lié, en particulier, à l'activité des animaux) favorisant la remobilisation des réserves à partir des tissus adipeux et responsable de la baisse de la fixation d'énergie au niveau global ; un accroissement de ce phénomène de remobilisation à partir des tissus adipeux en présence d'une insulinémie accrue, s'accompagnant, dans ces conditions d'un transfert d'une partie des acides gras libérés vers les muscles. 
Par ailleurs, chez les rats en libre consommation, l'ingestion de tricaproïne a exercé un effet opposé à celui que nous avons obtenu antérieurement (Chenat et al., 1976). Ces résultats contradictoires pourraient s'expliquer à partir des hypothèses émises ci-dessus et de l'amélioration de la fixation d'énergie et d'azote par les rats du lot témoin. Cependant, les effets de l'acide caproïque précédemment observés semblaient également avoir pour origine une augmentation de la glycémie et de la sécrétion d'insuline (Sunyer et al., 1969 ; Ambo et al., 1973 ; Caster et al., 1975). L'adjonction de saccharose à ce régime aurait donc dû, logiquement, renforcer cet effet et compenser ainsi celui obtenu avec le régime témoin. Mais, nos résultats antérieurs étaient obtenus sur une période de 21 jours. Or selon Caster et Bleecker (1975), l'élévation de la glycémie consécutive à l'ingestion d'acide caproïque n'intervient qu'après une période d'adaptation de 15 jours environ. La période expérimentale de 16 jours employée ici, aurait dans ces conditions été trop courte pour permettre l'obtention d'un accroissement du niveau d'insulinémie et de fixation d'énergie et d'azote. Et de fait, l'étude par calorimétrie indirecte des effets de l'ingestion de tricaproïne chez des rats de $100 \mathrm{~g}$ (Chenat et al., 1976) qui avait été réalisée dans des conditions voisines (animaux adaptés aux régimes pendant 10 jours et mesure lors des 5 jours suivants) n'avait pas révélé de différence entre l'utilisation d'un régime de ce type ou d'un régime témoin.

Enfin, l'absence d'effet au niveau de la carcasse entière, de la distribution du régime «Tri C6 » en 4 repas, peut être rapprochée de résultats semblables dans le cas d'agneaux préruminants (Walker et al., 1967) ou de porcelets (Sharma et al., 1973) recevant des régimes riches en graisses. Par contre on retrouve, comme dans le cas du régime témoin, l'opposition entre les effets observés au niveau global ou au niveau musculaire, ce qui confirme un effet spécifique du traitemenf « 4 repas » et reste en accord avec l'ensemble des hypothèses émises ci-dessus.

\section{Conclusion.}

Cette expérience est riche d'enseignements, le principal étant la mise en évidence du fait que la distribution des rations en 4 repas régulièrement espacés au cours des $24 \mathrm{~h}$ n'a pas d'effet sur l'utilisation globale de l'énergie et de l'azote d'un régime comportant de la tricaproïne.

Cependant, la distribution de la ration en quatre repas par jour modifie le métabolisme des animaux pour aboutir à un accroissement relatif du contenu en énergie et en azote des muscles des pattes.

Par ailleurs, l'obtention des résultats sensiblement différents de ceux observés précédemment soulève trois questions intéressantes :

- Quels peuvent être les rôles respectifs de la restriction alimentaire ou de la présence de saccharose dans les régimes sur l'accroissement relatif de l'énergie et de l'azote fixés chez les animaux "Témoins » recevant leurs rations en une seule distribution?

- Par quels mécanismes la distribution de 4 repas régulièrement espacés au cours de la journée aboutit-elle à des effets différents, et en particulier, à un transfert des réserves lipidiques vers les muscles, lorsque les rations sont davantage insulinogènes ? 
- Enfin, comment évolue le métabolisme des animaux lors de leur adaptation à un régime comportant de l'acide caproïque?

Reçu en novembre 1977.

Accepté en février 1978.

\section{References}

AMBO K., TAKAHASHI M., TSUNA T., 1973. Effect of feeding and infusion of short chain fatty acids on glucose levels in sheep. J. agric. Res., 24, 54-62.

AUROUSSEAU B., 1972. Utilisation énergétique des acides caprylique, laurique et myristique par le rat en croissance. Influence des restrictions alimentaires. Ann. Biol. onim. Biochim. Biophys., 12, 263-280.

AUROUSSEAU B., DE GROOT L., VERMOREL M., 1970. Etude comparée de l'utilisation énergétique de régimes riches en acide caprylique ou en acides gras insaturés. Ann. Biol. anim. Bioch. Biophys., 10, 703-706.

BOUVIER J. C., PEREZ J. M., AUROUSSEAU B., VERMOREL M., 1971. Influence de la fréquence des repas sur l'utilisation de l'énergie et de l'azote de la ration par le rat en croissance. 1 er Symp. Soc. Nufr. Dict. Lang. Franç. Paris, 16-18 sept. 1971, 312-315.

CASTER W. O., BLEECKER S., 1975. Hyperglycemic effect of amino compounds structurally related to caproate in rats. J. Nutr., 105, 308-310.

CASTER W. O., RESURRECTION A. V., CODY M., ANDREWS J. W. Jr., BARGMANN R., 1975, Diefary effects of the esters of butyric, caproic, caprylic, lauric, myristic, palmitic and stearic acids on food intake, weight gain, plasma glucose and tissue lipid in the male white rat. J. Nutr., 105, 676-687.

CHENAT M.-C., AUROUSSEAU B., VERMOREL M., 1976. Influence de l'acide caproïque sur l'utilisation de l'énergie et de l'azote ingérés par le rat en croissance en fonction de l'âge des animaux et de la teneur en azote des régimes. Ann. Biol. anim. Bioch. Biophys., 16, 603-622.

CHLOUVERAKIS C., 1967. Factors affecting the inhibitory action of insulin on lipolysis in a glucose free medium. Endocrinology, 81, 521-526.

FAIN J. N., KOYACEV V. P., SCOW R. O., 1966. Antilipolytic effect of insulin in isolated fat cells of the rat. Endocrinology, 78, 773-779.

GRIZARD J., PRUGNAUD J., PION R., 1974. Influence de l'alimentation par repas sur la composition corporelle et les teneurs sanguins en acides aminés libres chez le rat en croissance. C. R. Soc. Biol., 168, 738-743.

HORINO M., MACHLIN L. J., HERTELENDY F., KIPNIS D. M., 1968. Effect of short chain fatty acids on plasma insulin in ruminant and non ruminant species. Endocrinology, 83, 118-128.

KOVACEV V. P., SCOW R. O., 1966. Effect of hormones on fatty acid release by rat adipose tissue in vivo. Am. J. Physiol., 210, 1199-1208.

Mac RAE A. R., NICKEL I., SLINGER S. J., NEUDORFER T. S., 1974. Carcass composition and weight gain response of rats fed various carbohydrates at two dietary levels of carbohydrates and protein. Nutr. Metab., 17, 47-54.

MAHENDRA C. C., MILLER D. S., 1969. The effect of depletion repletion on calorie utilization. Proc. Nufr. Soc., 28, 7-24.

MILLER E. A., ALLEN D. O., 1973. Hormone stimulated lipolysis in isolated fat cells from «young » and "old" rats. J. Lip. Res., 14, 331-336.

NAKANO K., ASHIDA K., 1975. Possible intervention of insulin cyclic AMP and glucocorticoïds in protein sparing action of dietary carbohydrate in rats. J. Nutr., 105, 906-913.

PAWLAK M., PION R., 1968. Influence de la supplémentation des protéines de blé par des doses croissantes de lysine sur la teneur en acides aminés libres du sang et du muscle du rat en croissance. Ann. Biol. anim. Bioch. Biophys., 8, 517-530.

PAWLAK M., PION R., 1971. Influence de la teneur en isoleucine du régime sur la croissance ef la teneur en isoleucine libre du sang et du muscle. Ann. Biol. anim. Bioch. Biophys., 11, 505-510.

RIDER A. A., CHOW B. F., 1971. Body weight changes in rats rationed to there ad libitum feed intake. Nutr. Rep. Int., 3, 21-29. 
SHARMA V. O., YOUNG L. G., BROWN R. G., BUCHANAN-SMITH J., SMITH G. C., 1973. Effects of frequency of feeding on energy metabolism and body composition of young pigs. Can. J. Anim. Sci., 53, 157-164.

SUNYER F. X., HASHIM S. A., VAN ITALIE T. B., 1969. Insulin and ketone responses to ingestion of medium and long chain triglycerides in man. Diabetes, 181, 96-100.

VERMOREL M., KELLER J., 1967. Utilisation énergétique par le rat en croissance des principales céréales composant des régimes isoazotés et équilibrés en acides aminés. Ann. Zoofech., 16, 223-234.

WALKER D. M., COOK L. J., JAGUSH K. T., 1967. Nitrogen balance studies with milk fed lambs. 5. Effect of frequency of feeding. Br. J. Nutr., 21, 275-287.

WOOL I. G., STIREWALT W. S., KURIMARA K., LOW R. B., BAILLEY P., OYED, 1968. Mode of action of insulin in the regulation of protein biosynthesis in muscle. Rec. Progr. Horm. Res., 24, 139-213. 